\title{
Mass effects and internal space geometry in triatomic reaction dynamics
}

\author{
Tomohiro Yanao, ${ }^{*}$ Wang S. Koon, ${ }^{\dagger}$ and Jerrold E. Marsden ${ }^{\ddagger}$ \\ Control and Dynamical Systems, MC 107-81, California Institute of Technology, Pasadena, California 91125, USA
}

(Received 11 December 2005; published 10 May 2006)

\begin{abstract}
The effect of the distribution of mass in triatomic reaction dynamics is analyzed using the geometry of the associated internal space. Atomic masses are appropriately incorporated into internal coordinates as well as the associated non-Euclidean internal space metric tensor after a separation of the rotational degrees of freedom. Because of the non-Euclidean nature of the metric in the internal space, terms such as connection coefficients arise in the internal equations of motion, which act as velocity-dependent forces in a coordinate chart. By statistically averaging these terms, an effective force field is deduced, which accounts for the statistical tendency of geodesics in the internal space. This force field is shown to play a crucial role in determining mass-related branching ratios of isomerization and dissociation dynamics of a triatomic molecule. The methodology presented can be useful for qualitatively predicting branching ratios in general triatomic reactions, and may be applied to the study of isotope effects.
\end{abstract}

DOI: 10.1103/PhysRevA.73.052704 PACS number(s): 34.10.+x, 36.40.-c, 02.40. $-\mathrm{k}, 33.20 . \mathrm{Vq}$

\section{INTRODUCTION}

The configuration of atomic masses in a molecule is an important factor in a determination of branching ratios and rates of reactions of the molecule. In particular, the roles of mass configuration are of great interest in isotopic reactions. For example, branching ratios of the dissociation dynamics of triatomic hydrogen ion $\mathrm{H}_{3}^{+}$and its isotopomers, $\mathrm{D}_{2} \mathrm{H}^{+}$, $\mathrm{H}_{2} \mathrm{D}^{+}$, and $\mathrm{D}_{3}{ }^{+}$is the subject of extensive studies, both experimentally and theoretically (see Refs. [1-4]). Another challenging problem is the anomalous isotope effect in the formation and exchange reactions of ozone $\mathrm{O}_{3}$ (see Refs. [5-11]). Atomic masses are also found to play a crucial role in intramolecular vibrational-energy redistribution (IVR) in $\mathrm{N}_{2} \mathrm{O}$ reactions (see Ref. [12]). Even in these fundamental reactions, there still exist many interesting and unknown issues on the role of atomic masses, and a general framework to understand the roles of atomic masses should serve many purposes.

There is a useful framework that allows one to characterize the mass effect specifically for collinear triatomic reactions such as $A+B C \rightarrow A B+C$. This is the so-called skewed coordinate system framework $[13,14]$, in which the two interparticle distances $(A-B$ distance and $B-C$ distance) are chosen as internal coordinates and are properly embedded in a two-dimensional Euclidean space. As a result, the motion of the three atoms is expressed as a motion of a point mass (with constant mass) in this associated two-dimensional space. In this formalism, the mass effect is effectively included in the so-called skew angle between the two axes of the interparticle distances. It is well known that the skew angle plays an important role in determining energy conversions [15] and tunneling probabilities [16].

Mass effects are, without doubt, important in chemical reactions with higher degrees of freedom. However, it is not

\footnotetext{
*Electronic address: tyanao@cds.caltech.edu

${ }^{\dagger}$ Electronic address: koon@cds.caltech.edu

EElectronic address: marsden@cds.caltech.edu
}

necessarily a trivial problem how to characterize the mass effect for reaction dynamics with more than two degrees of freedom. The reaction-path Hamiltonian formalism (see Refs. $[17,18]$ ) may be useful for studying mass effects in high-dimensional reaction dynamics. In this formalism, the curvature of the reaction path can be the key to quantify the mass effects. One matter for concern is that this formalism employs the Eckart conditions $[19,20]$ for a formal separation of overall rotation and internal motion, which may disguise an important geometric properties of molecular internal space by approximating the internal space as flat (Euclidean) space (see Refs. [21,22]).

For an appropriate description of the mass effect in polyatomic molecules, correctly separating rotational and internal motion is crucial already at the first stage. This is because rotational and internal motion can couple dramatically, and a change in the mass distribution in a molecule plays an important role in the coupling process simply due to conservation of total angular momentum. In this respect, reduction theory from geometric mechanics $[23,24]$ and the associated gauge theory $[21,25-30]$ for $n$-body dynamics with rotational symmetry provides a quite universal basis for the study of the mass effect in molecular reaction dynamics. According to these theories, atomic masses are incorporated into the metric tensor of internal space in a way that is independent of the choice of body-fixed frame. Remarkably, the metric tensor is non-Euclidean for general three- and more-atom systems so that the internal space cannot be embedded into Euclidean space as in the case of collinear triatomic reactions. Therefore the characterization of the effect originating from the non-Euclidean geometry of the internal space is quite important for understanding the mass effect. It should also be noted that the metric tensor of the internal space is independent of the potential-energy surface, and is determined only by mass and shape of the system. Therefore kinematic effects arising from the non-Euclidean nature of internal space should be of universal importance in a variety of reactions.

It is crucial to choose a good coordinate system for the study of the geometry of the internal space. For triatomic systems, the so-called symmetrical coordinates [21,30-32] 
and various hyperspherical coordinates [34-39] are useful and have been extensively studied. An advantage of the symmetrical coordinates is that the metric tensor of internal space becomes remarkably simple (conformally flat) $[21,30]$, and thereby a fairly uniform treatment of the three internal coordinates is possible. For these reasons, we focus on these coordinates in this study. Its non-Euclidean nature indicates that trajectories in the internal space intrinsically possess "directionality" even without the influence of the potentialenergy surface. This directionality will be shown to be important in the branching processes in multichannel reactions. A heuristic way to characterize this directionality is to investigate the behavior of a cloud of geodesics, which are the motion in the absence of potential-energy surface. By comparing the directionality of multiple reaction pathways of interest in internal space with that of geodesics, one can identify geometrically preferable pathways out of them. This is a basic strategy of this study to characterize mass effect in branching ratio problems.

It is more desirable to characterize the statistical tendency of the geodesics analytically. A possible and concise way to achieve this goal is to investigate the terms, including connection coefficients, that originate from the non-Euclidean nature of the metric in the equations of motion. These terms serve as velocity-dependent forces in a coordinate chart, which of course influence the behavior of geodesics. Recently, this kind of velocity-dependent force, which is an internal centrifugal force associated with hyperangular motion (kinematic rotation), has been shown to induce a trapped motion around saddle points of few-atom clusters [40,41]. This trapped motion has also been accounted for from the perspective of curvature tensor [42]. On the other hand, this paper focuses on the problem of mass-related branching ratios in triatomic reactions, which is very important from the aspect of reaction rate theory. By statistically averaging the velocity-dependent forces, an averaged force field is deduced, which accounts for the tendency of behavior of geodesics. It will be shown that this force field does dominate mass-influenced branching ratios of isomerization and dissociation reactions of a triatomic molecule.

In this paper, we introduce a prototypical model for the study of the mass effect in Sec. II. The model is a triatomic Morse cluster, which, along with the Lennard-Jones clusters were extensively investigated by Berry and co-workers [43-46]. These clusters are also of interest from the perspective of the effect of angular momentum [47]. We modify the original Morse cluster by changing the mass parameters of atoms in order to study mass effect from a rather general view point. This system has two permutationally distinctive isomers connected by three reaction pathways, along which potential barrier height is the same while geometric properties of internal space are different as a result of the different mass configurations. Despite the same potential-energy barrier height, a significant difference arises in the probabilities of occurrence of reactions through respective pathways. A heuristic account for these mass-related branching ratios is first given in Sec. III in terms of geodesics in the internal space. We then deduce an averaged field of the velocitydependent force, which accounts for the statistical tendencies of geodesics in a coordinate chart. It is shown that this force field does dominate mass-influenced branching ratios of the isomerization dynamics. A reaction-path potential is finally introduced to quantify the effect of the averaged force. By a methodology similar to that in Sec. III, a qualitative account for the branching ratio of dissociation dynamics of the cluster is given in Sec. IV. The paper concludes in Sec. V with some remarks.

\section{MODEL SYSTEM AND ISOMERIZATION DYNAMICS}

\section{A. Modified $M_{3}$ cluster}

The model system we study in this paper is a triatomic cluster whose constituent particles interact through a pairwise Morse potential. The total angular momentum of the system is assumed to be zero throughout the paper in order to highlight the importance of the geometric structure of the internal space. The dimensionless Hamiltonian of the system is

$$
\frac{\mathcal{H}}{\varepsilon}=\frac{1}{2} m_{1} \dot{r}_{1}^{2}+\frac{1}{2} m_{2} \dot{\boldsymbol{r}}_{2}^{2}+\frac{1}{2} m_{3} \dot{\boldsymbol{r}}_{3}^{2}+\sum_{i<j}\left[e^{-2\left(d_{i j}-d_{0}\right)}-2 e^{-\left(d_{i j}-d_{0}\right)}\right],
$$

where $\boldsymbol{r}_{i}=\left(r_{i x}, r_{i y}, r_{i z}\right)^{T}(i=1,2,3)$ is the three-dimensional position vector of each atom. The parameters $\left(m_{1}, m_{2}, m_{3}\right)$ are the mass parameters of respective atoms. The triatomic Morse and Lennard-Jones clusters with equal masses, $\left(m_{1}, m_{2}, m_{3}\right)=(1,1,1)$, are extensively studied by many authors [43-47]. This study focuses on the system with $\left(m_{1}, m_{2}, m_{3}\right)=(1,1,0.1)$, two heavy atoms and one light atom, for the study of the effect of different mass configurations in multi-channel reactions. Because of the mass difference, we call this system "modified" $M_{3}$ cluster. The parameter $\varepsilon$ represents the depth of the Morse potential and $d_{i j}$ is the interparticle distance between the $i$ th and $j$ th atoms. The parameter $d_{0}$, which corresponds to the equilibrium distance of the pairwise Morse potential, controls the Hamiltonian and we set this parameter to $d_{0}=6.0$, which provides a potential topography similar to that of the Lennard-Jones potential. In what follows, our numerical results are presented in the absolute units.

The isomerization scheme of the modified $M_{3}$ cluster is summarized in Fig. 1. This cluster has two local equilibrium structures (isomers) that correspond to permutationally distinctive equilateral triangles whose potential energy is $V=-3.00 \varepsilon$. These two structures, (a) and (b) in Fig. 1, are mutually distinctive since motion of the system is confined to a plane because of the conditions of vanishing total angular momentum. The system has three saddle points with potential energy $V \approx-2.005 \varepsilon$, which correspond to the collinear configurations as in Fig. 1. These collinear saddle structures are also permutationally distinctive. If the system has high enough energy, the structural isomerization reactions can occur through the three reaction channels corresponding to the three saddle points. These three channels are classified into two equivalent channels (channel 1 and channel 2) and one different kind of channel (channel 3). Channels 1 and 2 have the same mass configuration of heavy-heavy-light, while 


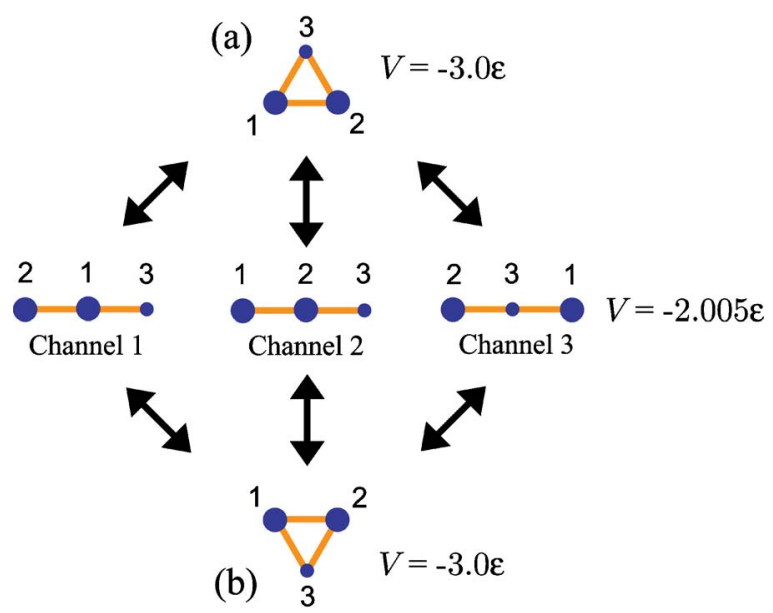

FIG. 1. (Color online) The isomerization scheme of the modified $M_{3}$ cluster. Atoms 1 and 2 have the same mass parameter $m_{1}=m_{2}=1$, and atom 3 has the mass parameter $m_{3}=0.1$. This cluster has two locally equilibrium structures, (a) and (b), which are equilateral triangle and permutationally distinct. The potential energy of these two structures is $V=-3.00 \varepsilon$. The cluster has three permutationally distinctive saddle structures, which are collinear, while the potential energy of these saddle structures is $V=-2.005 \varepsilon$. The number of the reaction channel is defined by the number of the atom in the middle at the saddle structure. Channel 1 and channel 2 are essentially equivalent and channel 3 is qualitatively different from other two channels.

channel 3 has the mass configuration of heavy-light-heavy. Note that the potential-barrier height for these three channels are exactly the same since the three particles interact equally through the Morse potential. The difference among the three channels lies in the way of changing mass distribution during the isomerization reactions.

\section{B. A coordinate system for the study of triatomic reactions}

Here, we introduce an internal coordinate system for the study of reaction dynamics of the modified $M_{3}$ cluster. In order to eliminate the translational degrees of freedom, we first define the mass-weighted Jacobi vectors as

$$
\begin{gathered}
\boldsymbol{\rho}_{1}=\sqrt{\mu_{1}}\left(\boldsymbol{r}_{1}-\boldsymbol{r}_{2}\right), \quad \mu_{1}=\frac{m_{1} m_{2}}{m_{1}+m_{2}}, \\
\boldsymbol{\rho}_{2}=\sqrt{\mu_{2}}\left(\frac{m_{1} \boldsymbol{r}_{1}+m_{2} \boldsymbol{r}_{2}}{m_{1}+m_{2}}-\boldsymbol{r}_{3}\right), \quad \mu_{2}=\frac{\left(m_{1}+m_{2}\right) m_{3}}{m_{1}+m_{2}+m_{3}},
\end{gathered}
$$

where $\mu_{1}$ and $\mu_{2}$ are the reduced masses. Then, the internal coordinates that we focus on in this paper are defined as

$$
\begin{gathered}
w_{1}=\left|\boldsymbol{\rho}_{1}\right|^{2}-\left|\boldsymbol{\rho}_{2}\right|^{2}, \\
w_{2}=2 \boldsymbol{\rho}_{1} \cdot \boldsymbol{\rho}_{2}, \\
w_{3}= \pm 2\left|\boldsymbol{\rho}_{1} \times \boldsymbol{\rho}_{2}\right| .
\end{gathered}
$$

Since the internal degree of freedom of the triatomic system is three, there are three internal (shape) coordinates (a)
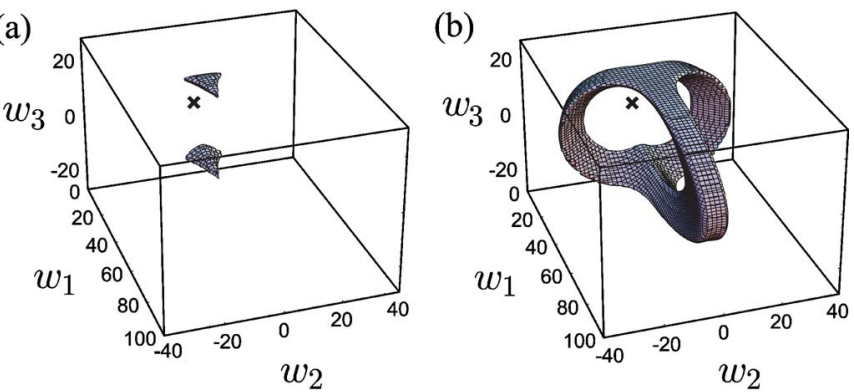

FIG. 2. (Color online) Equipotential-energy surfaces (Hill regions) in the internal space, at (a) $V=-2.2 \varepsilon$ and (b) $V=-1.4 \varepsilon$. The cross mark represents the origin, where $\left(w_{1}, w_{2}, w_{3}\right)=(0,0,0)$.

$\left(w_{1}, w_{2}, w_{3}\right)$. These coordinates are known as symmetrical coordinates [21,30-32] since the kinetic-energy expression in terms of these three variables becomes fairly symmetric as will be shown in Sec. III. All the internal coordinates range from $-\infty$ to $+\infty$, and the sign of $w_{3}$ specifies the permutational isomers [33], (a) and (b) in Fig. 1.

In Fig. 2, the global structure of the internal space is shown. The two equilibrium points (minima of the potential energy) of the cluster are located at $\left(w_{1}, w_{2}, w_{3}\right)$ $=(15.429,0, \pm 13.607)$ in these coordinates. Figure 2(a) shows equipotential energy surfaces at $V=-2.2 \varepsilon$. There exist two closed regions around the two equilibrium points. Classical trajectories with total energy $E=-2.2 \varepsilon$ can move around in the interior regions of the equipotential surfaces, which are called Hill regions. Since the two Hill regions are separated in Fig. 2(a), isomerization reaction is impossible at this energy. Figure 2(b) shows the equipotential surface (Hill region) at total energy $E=-1.4 \varepsilon$. At this energy, the two isomer regions are connected through the three "handlelike" structures, and thereby isomerization reaction is possible. Figure 3 shows the steepest descent paths connecting the two equilibrium configurations via the three collinear saddle configurations. These paths can be roughly regarded as the skel-

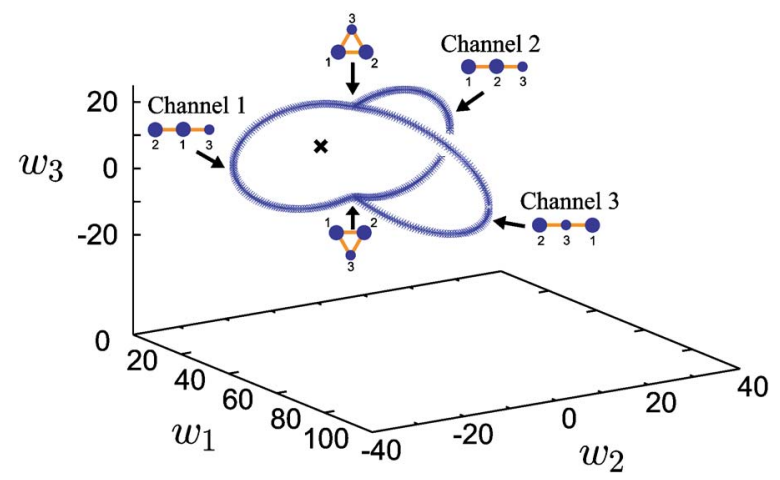

FIG. 3. (Color online) The steepest descent paths of the potential energy in the internal space that connect saddle points and local minima and the corresponding structures of the cluster are shown. The two equilibrium points of the cluster are at $\left(w_{1}, w_{2}, w_{3}\right)$ $=(15.429,0, \pm 13.607)$. The saddle points for channels 1,2 , and 3 are located at $\left(w_{1}, w_{2}, w_{3}\right)=(10.277,-23.548,0), \quad\left(w_{1}, w_{2}, w_{3}\right)$ $=(10.277,23.548,0)$, and $\left(w_{1}, w_{2}, w_{3}\right)=(71.939,0,0)$ respectively. The cross mark represents the origin, $\left(w_{1}, w_{2}, w_{3}\right)=(0,0,0)$. 
eton of the reaction handles in Fig. 2. The saddle point for channel 1 is located at $\left(w_{1}, w_{2}, w_{3}\right)=(10.277,-23.548,0)$ while that for channel 2 is located at $\left(w_{1}, w_{2}, w_{3}\right)$ $=(10.277,23.548,0)$. The saddle point for channel 3 is located at $\left(w_{1}, w_{2}, w_{3}\right)=(71.939,0,0)$. Since the potential energy of these three saddle points is the same, reaction through these three channels becomes energetically possible at the same energy.

The Hill regions in Fig. 2 and the paths in Fig. 3 have reflection symmetry with respect to the $w_{1}-w_{2}$ plane and the $w_{1}-w_{3}$ plane. The reflection symmetry with respect to the $w_{1}-w_{2}$ plane is due to the permutational symmetry between isomer (a) and isomer (b) in Fig. 1. The reflection symmetry with respect to the $w_{1}-w_{3}$ plane is due to the symmetry with respect to the permutation of atom 1 and atom 2 . This property is consistent with the fact that channel 1 and channel 2 are equivalent and only channel 3 is qualitatively different from other two channels. It should be noted that if the three atoms have the same mass, the three reaction pathways (handlelike structures) in Fig. 2 have the symmetric structure surrounding the origin $\left(w_{1}, w_{2}, w_{3}\right)=(0,0,0)$. Specialty of the mass of the third atom brings about the deformation of Hill region as in Fig. 2. This deformed structure of Hill region will be the key in characterizing mass effect later.

\section{Reaction frequencies of isomerization: Numerical study}

This section summarizes the results of numerical experiments on reaction frequencies of isomerization dynamics of the modified $M_{3}$ cluster, and raise an important issue to be investigated in the following section.

As a measure of reaction frequency (branching ratio) of each channel, one might want to count the frequency that the system crosses each collinear configuration as dividing surface per unit time. However, this definition of reaction frequency is not necessarily appropriate since the system often recrosses the collinear configurations before getting to the vicinities of the equilibrium points. Therefore, in order to avoid overcounting due to the recrossing as much as possible, we introduce more strict conditions for isomerization as follows: We count the total number $N_{i}$ that the system passes through each "handle" in Fig. 2(b) corresponding to each channel $i(i=1,2,3)$ in many long time trajectories. In this computation, we count only the event that the system enters into one of the handles at one side and subsequently gets out of the handle at the other side without returning to the entering side. In other words, we count the total number that the system completes reaction through each channel. Region of each handle in internal space is defined in terms of the three inter particle distances, $d_{12}, d_{13}, d_{23}$, as

$$
\begin{array}{ll}
\text { handle 1: } & d_{23}>d_{12}, d_{13}, \\
\text { handle 2: } & d_{13}>d_{12}, d_{23}, \\
\text { handle 3: } & d_{12}>d_{13}, d_{23},
\end{array}
$$

where the number of each handle is concomitant with that of the reaction channel.
TABLE I. Reaction frequency through each channel for the 3000 trajectories whose initial conditions are randomly sampled and whose integration time is 5000 absolute units.

\begin{tabular}{crrr}
\hline \hline Energy $(\varepsilon)$ & Channel 1 & Channel 2 & Channel 3 \\
\hline-1.9 & 32668 & 32266 & 14725 \\
-1.8 & 82527 & 81956 & 31061 \\
-1.7 & 127671 & 128384 & 46004 \\
-1.6 & 173037 & 172683 & 51657 \\
-1.5 & 209088 & 208011 & 55604 \\
-1.4 & 241208 & 241327 & 56061 \\
-1.3 & 266156 & 266622 & 56778 \\
-1.2 & 291007 & 290613 & 54459 \\
-1.1 & 306109 & 306601 & 57081 \\
\hline \hline
\end{tabular}

Table I shows the results of the total reaction frequency $N_{i}$ through each channel $i(i=1,2,3)$ for the 3000 trajectories whose initial conditions are randomly sampled. At the same time, Fig. 4 shows the normalized reaction frequency (probability) computed by

$$
f_{i}=\frac{N_{i}}{N_{1}+N_{2}+N_{3}} \times 100 \quad(i=1,2,3) .
$$

Since channel 1 and channel 2 are equivalent, frequencies for these two channels are almost the same. It is evident from Fig. 4 and Table I that reaction through channel 3 is much less frequent than those through channel 1 and channel 2. In other words, the system prefers the reaction through heavyheavy-light configurations rather than heavy-light-heavy configurations. Furthermore, we see that the reaction through channel 3 becomes much less frequent as the energy of the system increases.

The significant bias in the reaction frequency seen in Fig. 4 cannot be explained simply by the potential-energy barrier height since the barrier height for the three channels are the same. Instead, the bias must be related to the mass configuration. This result indicates that there must be a certain

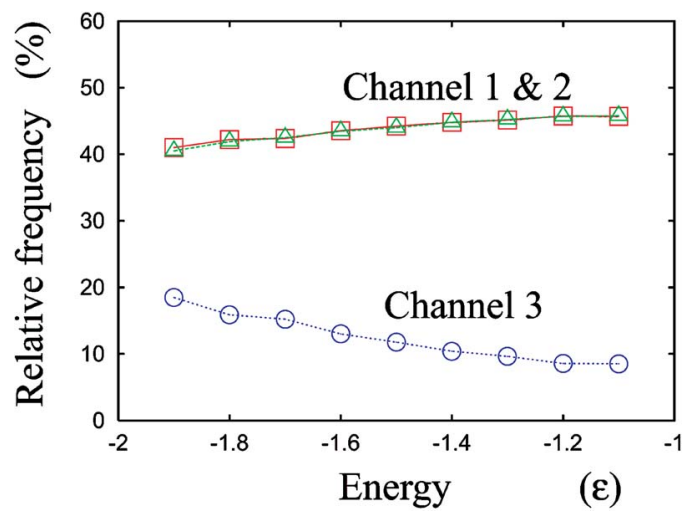

FIG. 4. (Color online) Energy dependence of relative reaction frequency for channel 1 (open squares), channel 2 (open triangles), and channel 3 (open circles). The frequencies are normalized among the three channels based on Eq. (10). 
mechanism that makes the reaction through channel 3 harder than reactions through channel 1 and 2 . Therefore we need to look further into the geometric properties of the internal space that are related to mass effects. In the next section, we focus on those geometric issues to account for the bias found in the reaction frequency in Fig 4. Extracting a mechanism that makes the reaction through channel 3 difficult is the goal of the next section.

\section{GEOMETRIC CHARACTERIZATION OF MASS EFFECT IN ISOMERIZATION DYNAMICS}

\section{A. Separation of rotation and internal motion}

For a correct discussion of the mass effect in the dynamics of polyatomic molecules, a correct separation of rotations is crucial. This section summarizes the separation procedure from a general point of view. The total kinetic energy of the system is initially expressed as

$$
K=\frac{1}{2} \sum_{i=1}^{2} \dot{\boldsymbol{\rho}}_{i} \cdot \dot{\boldsymbol{\rho}}_{i}
$$

where overall translational degrees of freedom are automatically eliminated by using the Jacobi vectors $\left\{\boldsymbol{\rho}_{i}\right\}$ defined in Eq. (3). According to reduction theory $[23,24]$ or the associated gauge theoretic point of view $[21,25-30]$, the total kinetic energy Eq. (11) can be rewritten generally as

$$
K=\frac{1}{2} \boldsymbol{J}^{T} \mathbf{M}^{-1} \boldsymbol{J}+\frac{1}{2} g_{\mu \nu} \dot{q}^{\mu} \dot{q}^{\nu},
$$

where $\boldsymbol{J}$ is the total angular momentum, $\mathrm{M}$ is moment of inertia tensor, $\left\{q^{\mu}\right\}$ are arbitrary internal coordinates, and $g_{\mu \nu}$ is the gauge-invariant metric tensor of internal space that is independent of the choice of body frame. In Eq. (12), the summation with respect to the Greek indices $\mu$ and $\nu$ over all the internal degrees of freedom of the system is implicitly assumed. The advantage of the expression Eq. (12) is that both the first and the second terms in the right-hand side are invariant under a change in the choice of body frame, and the first term vanishes identically if the total angular momentum is zero $(\boldsymbol{J}=\mathbf{0})$, which is the case of the present study. Therefore the Lagrangian for the system with zero total angular momentum is

$$
L=\frac{1}{2} g_{\mu \nu} \dot{q}^{\mu} \dot{q}^{\nu}-V\left(\left\{q^{\mu}\right\}\right),
$$

where we have restricted the potential function $V$ to be a function only of the internal coordinates $\left\{q^{\mu}\right\}$, which is the case of our cluster. Since the metric tensor $g_{\mu \nu}$ is generally non-Euclidean for three-body and more-body systems, equations of motion derived from the Lagrangian Eq. (13) are in the form

$$
g_{\mu \nu}\left(\ddot{q}^{\nu}+\Gamma_{\kappa \lambda}^{\nu} \dot{q}^{\kappa} \dot{q}^{\lambda}\right)=-\frac{\partial V}{\partial q^{\mu}},
$$

where the connection coefficients (Christoffel symbols) $\Gamma_{\kappa \lambda}^{\nu}$ are defined by

$$
\Gamma_{\kappa \lambda}^{\nu}=\frac{1}{2} g^{\nu \mu}\left(\frac{\partial g_{\mu \kappa}}{\partial q^{\lambda}}+\frac{\partial g_{\mu \lambda}}{\partial q^{\kappa}}-\frac{\partial g_{\kappa \lambda}}{\partial q^{\mu}}\right) .
$$

Since the original atomic masses $\left\{m_{i}\right\}$ are incorporated into the non-Euclidean metric tensor $g_{\mu \nu}$ and the internal coordinates, the terms including the Christoffel symbols in the lefthand side of Eq. (14) are important to characterize the mass effect. It should be noted that these terms play a role of velocity-dependent forces in a coordinate chart like Fig. 2. Therefore the next step is to analyze the properties of these terms using the internal coordinate system $\left(w_{1}, w_{2}, w_{3}\right)$.

\section{B. Metric tensor of internal space and equations of motion}

It is shown in Refs. [21,30] that the representation of the internal space metric tensor in the coordinates $\left(w_{1}, w_{2}, w_{3}\right)$ is given by

$$
\left(g_{\mu \nu}\right)=\left(\begin{array}{ccc}
\frac{1}{4 w} & 0 & 0 \\
0 & \frac{1}{4 w} & 0 \\
0 & 0 & \frac{1}{4 w}
\end{array}\right), \quad w=\sqrt{w_{1}^{2}+w_{2}^{2}+w_{3}^{2}},
$$

which is a conformally flat metric. This conformally flat nature of the metric, as revealed in the coordinates $\left(w_{1}, w_{2}, w_{3}\right)$, makes it possible to treat the coordinates in a symmetrical manner. This is the advantage of this internal coordinate system. According to Eq. (13), the Lagrangian for the triatomic system with zero-angular momentum is

$$
\mathcal{L}=\frac{1}{2}\left(\frac{1}{4 w}\right) \dot{w}_{1}^{2}+\frac{1}{2}\left(\frac{1}{4 w}\right) \dot{w}_{2}^{2}+\frac{1}{2}\left(\frac{1}{4 w}\right) \dot{w}_{3}^{2}-V\left(w_{1}, w_{2}, w_{3}\right) .
$$

The classical equations of motion derived from this Lagrangian are

$$
\begin{aligned}
& \frac{1}{4 w} \ddot{w}_{1}-\frac{w_{1}}{8 w^{3}} \dot{w}_{1}^{2}+\frac{w_{1}}{8 w^{3}} \dot{w}_{2}^{2}+\frac{w_{1}}{8 w^{3}} \dot{w}_{3}^{2}-\frac{w_{2}}{4 w^{3}} \dot{w}_{1} \dot{w}_{2}-\frac{w_{3}}{4 w^{3}} \dot{w}_{1} \dot{w}_{3} \\
& =-\frac{\partial V}{\partial w_{1}}, \\
& \frac{1}{4 w} \ddot{w}_{2}+\frac{w_{2}}{8 w^{3}} \dot{w}_{1}^{2}-\frac{w_{2}}{8 w^{3}} \dot{w}_{2}^{2}+\frac{w_{2}}{8 w^{3}} \dot{w}_{3}^{2}-\frac{w_{1}}{4 w^{3}} \dot{w}_{2} \dot{w}_{1}-\frac{w_{3}}{4 w^{3}} \dot{w}_{2} \dot{w}_{3} \\
& =-\frac{\partial V}{\partial w_{2}}, \\
& \frac{1}{4 w} \ddot{w}_{3}+\frac{w_{3}}{8 w^{3}} \dot{w}_{1}^{2}+\frac{w_{3}}{8 w^{3}} \dot{w}_{2}^{2}-\frac{w_{3}}{8 w^{3}} \dot{w}_{3}^{2}-\frac{w_{2}}{4 w^{3}} \dot{w}_{3} \dot{w}_{2}-\frac{w_{1}}{4 w^{3}} \dot{w}_{3} \dot{w}_{1} \\
& =-\frac{\partial V}{\partial w_{3}} .
\end{aligned}
$$

There are five terms quadratic in the velocity components 
$\left\{\dot{w}_{i}\right\}$ on the left-hand sides of Eqs. (18)-(20) (from the second to the sixth terms on the left-hand sides). These terms correspond to the terms involving the connection coefficients in Eq. (14) and can be regarded as velocity-dependent "forces" at each point in the coordinate chart. Therefore it is important to extract essential properties of these terms that have originated from the non-Euclidean nature of the metric.

\section{Averaged force field and geodesics}

In discussing statistical properties of reaction dynamics such as the reaction frequency or the branching ratio, it is important to characterize how the velocity-dependent terms (forces) in Eqs. (18)-(20) affect the behavior of ensemble of trajectories in the internal space. If the distribution of velocity vectors of the ensemble of trajectories can be assumed to be sufficiently stochastic at each point in the internal space, it is reasonable to evaluate microcanonical averages for the quadratic factors of the velocity components at each point in internal space such as $\left\langle\dot{w}_{i} \dot{w}_{j}\right\rangle$. Since the generalized momenta conjugate to $\left(w_{1}, w_{2}, w_{3}\right)$ are

$$
p_{i}=\frac{\partial \mathcal{L}}{\partial \dot{w}_{i}}=\frac{\dot{w}_{i}}{4 w} \quad(i=1,2,3),
$$

microcanonical averages for the quadratic factors $\dot{w}_{i} \dot{w}_{j}(i, j$ $=1,2,3)$ at each point in internal space are computed by

$$
\left\langle\dot{w}_{i} \dot{w}_{j}\right\rangle=\frac{(4 w)^{2}}{\Omega(K)} \int_{-\infty}^{+\infty} p_{i} p_{j} \delta\left(K-2 w\left(p_{1}^{2}+p_{2}^{2}+p_{3}^{2}\right)\right) d p_{1} d p_{2} d p_{3},
$$

where $\delta(\cdots)$ is the delta function, $K=K\left(w_{1}, w_{2}, w_{3}\right)$ is the kinetic energy at each point in the internal space, and $\Omega(K)$ is the normalization factor,

$$
\Omega(K)=\int_{-\infty}^{+\infty} \delta\left(K-2 w\left(p_{1}^{2}+p_{2}^{2}+p_{3}^{2}\right)\right) d p_{1} d p_{2} d p_{3} .
$$

Equation (22) yields for the quadratic diagonal terms

$$
\left\langle\dot{w}_{1}^{2}\right\rangle=\left\langle\dot{w}_{2}^{2}\right\rangle=\left\langle\dot{w}_{3}^{2}\right\rangle=\frac{8 w K}{3} .
$$

These equalities reproduce the equipartition law for kinetic energy at each point in internal space,

$$
\left\langle\frac{1}{2}\left(\frac{1}{4 w}\right) \dot{w}_{1}^{2}\right\rangle=\left\langle\frac{1}{2}\left(\frac{1}{4 w}\right) \dot{w}_{2}^{2}\right\rangle=\left\langle\frac{1}{2}\left(\frac{1}{4 w}\right) \dot{w}_{3}^{2}\right\rangle=\frac{K}{3} .
$$

As for the cross terms, Eq. (22) yields

$$
\left\langle\dot{w}_{i} \dot{w}_{j}\right\rangle=0 \quad(\text { for } i \neq j) .
$$

This is because the integrand in Eq. (22) is odd with respect to $p_{i}$ and $p_{j}$ if $i \neq j$. Applying Eqs. (24) and (26) to the velocity-dependent terms in Eqs. (18)-(20), one finally obtains an averaged field of the velocity dependent force as
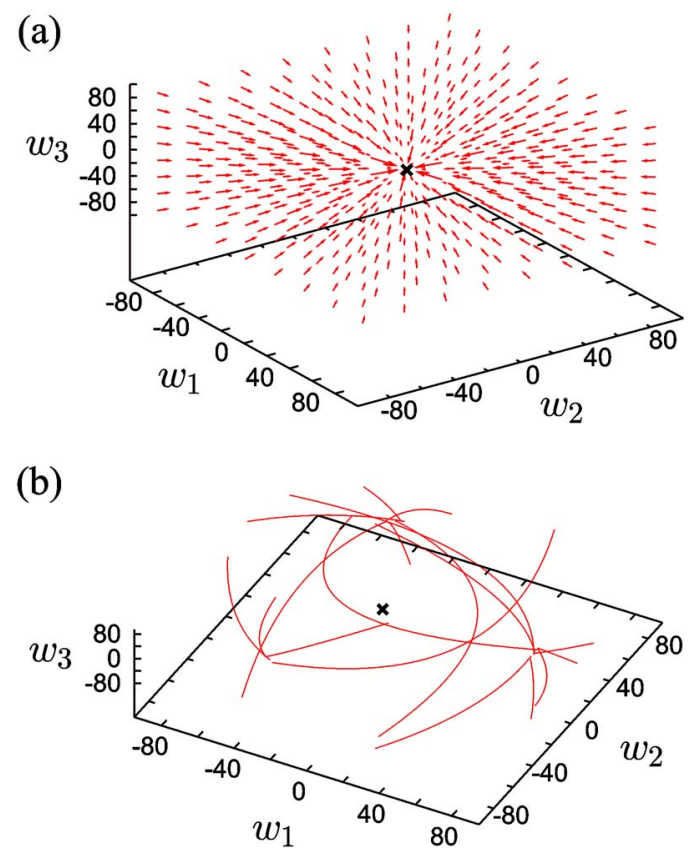

FIG. 5. (Color online) (a) Averaged field of the velocitydependent force in the internal space [Eq. (27)]. The kinetic energy $K$ is set to be constant over the internal space. Length of arrows reflects the strength of the force at each point. (b) Geodesics in the internal space started at the three points around the origin of the space with random initial velocities. Cross marks in (a) and (b) represent the origin, $\left(w_{1}, w_{2}, w_{3}\right)=(0,0,0)$.

$$
\left(-\frac{K w_{1}}{3 w^{2}},-\frac{K w_{2}}{3 w^{2}},-\frac{K w_{3}}{3 w^{2}}\right) .
$$

This force field should effectively influence the behavior of ensemble of trajectories in the internal space.

In order to study the effects of the averaged force field of Eq. (27), we first analyze the behavior of the geodesics in the internal space. Physically, the geodesics correspond to the motions of three free particles with zero total angular momentum. They are the trajectories under no influence of potential-energy surface in internal space, whose equations of motion are obtained by setting the right-hand sides of Eqs. (18)-(20) to zero. Since there is no potential-energy surface, the kinetic energy $K$ is constant over the internal space for the ensemble of geodesic motions with the same energy $E=K$. In Fig. 5(a), the field of the averaged force of Eq. (27) is shown by setting the kinetic energy $K$ constant all over the internal space. This averaged force field looks like a "central force field" that points to the origin $\left(w_{1}, w_{2}, w_{3}\right)=(0,0,0)$. In the figure, length of each arrow represents the magnitude of the force at each point. The averaged force field tends to be significant in the vicinity of the origin of the space. Figure 5(b) shows the behavior of geodesics started at the three points around the origin with random initial velocities. Observe that the geodesics tend to be curved to encircle the origin. This behavior confirms that the central force field should effectively exist in the internal space.

It is insightful to compare the directionality of geodesics with that of the reaction pathways of our triatomic cluster. 


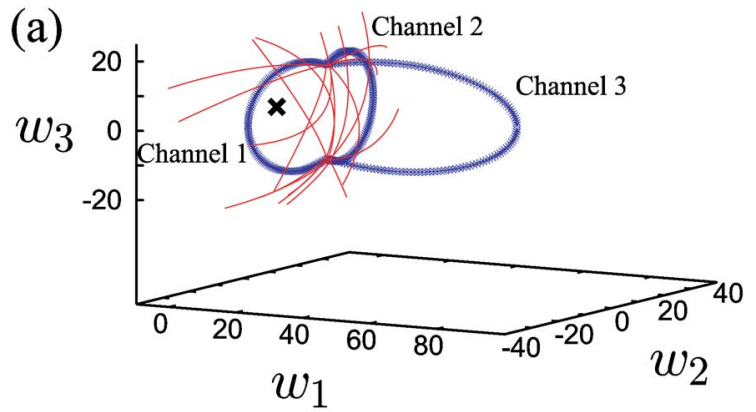

(b)

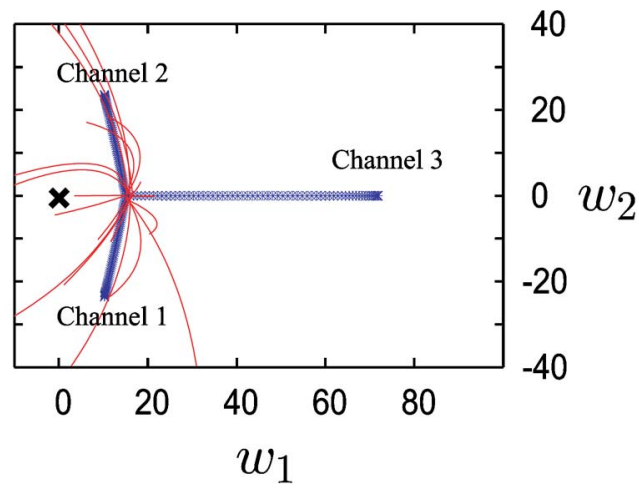

FIG. 6. (Color online) (a) Geodesics in the internal space randomly started at the two points, $\left(w_{1}, w_{2}, w_{3}\right)=(15.429,0, \pm 13.607)$, which correspond to the equilibrium points of the modified $M_{3}$ cluster. For comparison, the steepest descent paths in Fig. 3 are shown with the bold curves in the same figure. (b) is a top view of (a). Cross marks in (a) and (b) represent the origin, $\left(w_{1}, w_{2}, w_{3}\right)$ $=(0,0,0)$.

Figures 6(a) and 6(b) show the geodesics started at the vicinities of the two equilibrium points of the cluster, which are $\left(w_{1}, w_{2}, w_{3}\right)=(15.429,0, \pm 13.607)$. The initial velocities of the geodesics are prepared randomly. Figure 6(b) is a top view of Fig. 6(a). Because of the central force field mentioned above, geodesics have a constant tendency to be curved to encircle the origin. As a result, geodesics are diverted from the direction of the reaction pathway of channel 3 , and have preference to go in the direction of channel 1 and channel 2. This fact means that the motion of the three free particles with zero total angular momentum already have the strong preference to channel 1 and channel 2 even without the interaction force. This preference of motion of geodesics is the geometric origin of the significant difference in reaction frequencies observed in Fig. 4.

\section{Dynamical barrier and reaction-path potential}

We next discuss the full dynamics with potential-energy surface. Under the influence of potential energy $V\left(w_{1}, w_{2}, w_{3}\right)$, the kinetic energy $K$ in Eq. (27) is a function of the internal coordinates as $K=E-V\left(w_{1}, w_{2}, w_{3}\right)$. Figures 7 (a) and 7(b) show the averaged field of the velocitydependent force in the Hill region at $E=-1.4 \varepsilon$. The averaged force field tends to be strong in the vicinity of the equilibrium points (bottom of potential energy) since the kinetic energy $K$ in Eq. (27) becomes large there. Directionality of
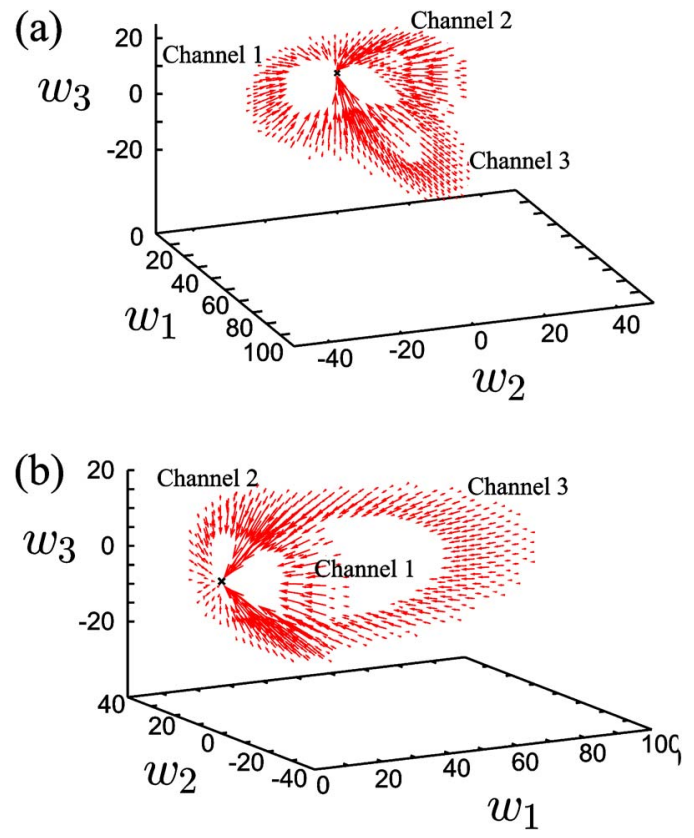

FIG. 7. (Color online) (a) Averaged field of the velocitydependent force in the Hill region at $E=-1.4 \varepsilon$. (b) The same field as in (a) seen from a different view point.

the field in Fig. 7 is essentially the same as that in Fig. 5. That is, the force field in the Hill region points to the origin of the space. As a result, the averaged force field works to block trajectories to get into the reaction pathway of channel 3 in the vicinity of the two equilibrium points. In this way, the averaged field of the velocity-dependent force works as a dynamical barrier for channel 3. On the other hand, the averaged force field works rather perpendicularly to the pathways of channel 1 and 2 . As a result, the force field does not hinder the reactions through channel 1 and 2 as significantly.

To quantify the effect of the averaged force field, a "reaction-path potential" is introduced next, which is defined as the sum of the potential and the work necessary to bring the system against the averaged force along the path. For simplicity, the steepest descent path in Fig. 3 is chosen as the reaction path. According to the metric Eq. (16), arc length $s$ of the path is computed as

$$
s=\int \sqrt{\frac{1}{4 w}\left(\frac{d w_{1}}{d \sigma}\right)^{2}+\frac{1}{4 w}\left(\frac{d w_{2}}{d \sigma}\right)^{2}+\frac{1}{4 w}\left(\frac{d w_{3}}{d \sigma}\right)^{2}} d \sigma,
$$

where $\sigma$ is a parameter of the path. Then the reaction-path potential is defined as the sum of the original potential and the line integral of the negative of the averaged force field:

$$
\begin{aligned}
V_{\text {path }}(s)= & V(s)+\int_{\text {path }} \frac{E-V\left(w_{1}, w_{2}, w_{3}\right)}{3 w^{2}} \\
& \times\left(w_{1} \frac{d w_{1}}{d s}+w_{2} \frac{d w_{2}}{d s}+w_{3} \frac{d w_{3}}{d s}\right) d s,
\end{aligned}
$$

where the integration is along a reaction path. Figures 8(a) and 8 (b) show the original potential and the reaction-path 
(a)

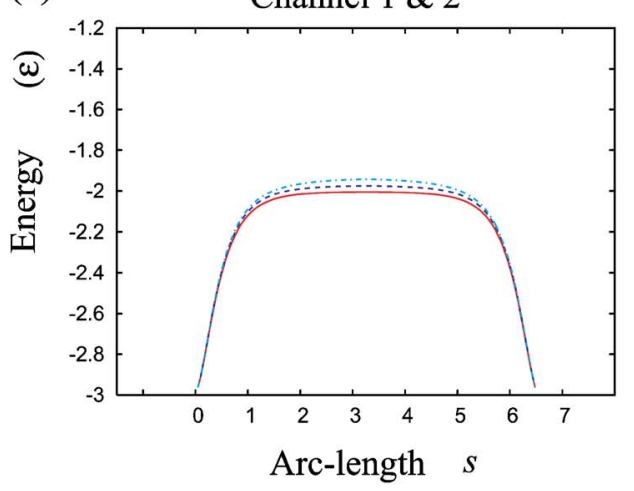

(b)

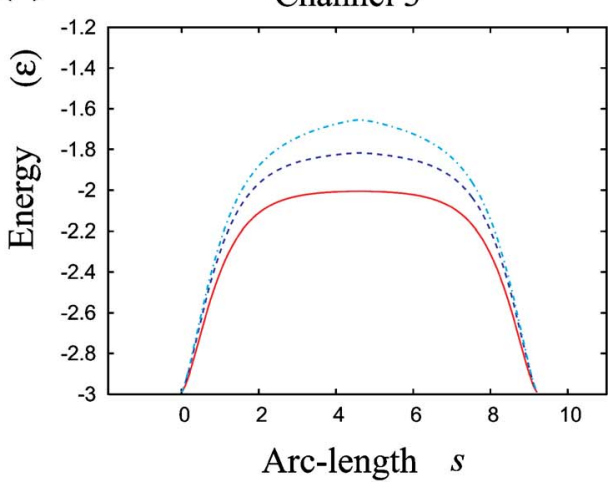

FIG. 8. (Color online) The reaction-path potential along the steepest descent paths in Fig. 3. (a) is for channels 1 and 2, and (b) is for channel 3. Solid curves are the original potential for the respective channels. The broken curves and the dotted curves are the reaction-path potential at total energy $E=-1.8 \varepsilon$ and $E=-1.4 \varepsilon$, respectively. potential along the reaction paths for respective channels. The height of the original potential energy barrier is the same in (a) (channel 1 and channel 2) and (b) (channel 3). In channels 1 and 2, the energy dependence of the reaction-path potential is very weak. On the other hand, in channel 3 , the reaction-path potential becomes much higher as the energy increases. This explains why the reaction through channel 3 becomes much less frequent than the reactions through channel 1 and channel 2 as the energy increases. In this way, the influence of the averaged force field on the reaction dynamics of each channel is characterized by the reaction-path potential.

\section{DISSOCIATION DYNAMICS}

\section{A. Dissociation channels and branching ratio}

We next investigate the role of the averaged field of velocity-dependent force in dissociation dynamics of the modified $M_{3}$ cluster with zero total angular momentum. All the system parameters are the same as the ones defined in Sec. II, and thereby mass parameters are $\left(m_{1}, m_{2}, m_{3}\right)$ $=(1,1,0.1)$. If the total internal energy is higher than $E=-1.0 \varepsilon$ and lower than $E=0.0 \varepsilon$, the triatomic cluster can break up into a single atom and a diatomic system. If the energy is above $E=0.0 \varepsilon$, breaking up into three single atoms is also energetically possible, but we do not consider this energy range in this paper. In the dissociation reactions in which the system breaks up into a single atom and a diatomic system, there are three possible channels as depicted in Fig. 9. The dissociation channels are named by the labels of the dissociating single atom. Since the mass of atom 1 and that of atom 2 are the same, dissociation channels 1 and 2 are essentially equivalent, and only the dissociation channel 3 is different.

The main interest of this study is the branching ratio among the three dissociation channels. This branching ratio is of interest in view of the dissociation dynamics of one of the isotopomers of triatomic hydrogen ion, $\mathrm{D}_{2} \mathrm{H}^{+}[2,4]$. Note that both our modified $M_{3}$ cluster and $\mathrm{D}_{2} \mathrm{H}^{+}$are composed of the two relatively heavy atoms and one light atom. We randomly prepared 50000 initial conditions around the equilibrium point (equilateral triangle) of the modified $M_{3}$ cluster and ran respective trajectories until they dissociate, and counted the branching ratio among the three dissociation channels. At total energy $E=-0.5 \varepsilon$, the 50000 trajectories are branched into the three dissociation channels with the ratio

Ch. 1:Ch. 2:Ch. 3

$$
=12581(25.162 \%): 12489(24.978 \%): 24930(49.86 \%) \text {. }
$$

Since the dissociation channels 1 and 2 are essentially equivalent, the percentage for these two channels are almost the same. It turned out that the dissociation channel 3 is much favored compared with other two channels (roughly twice). This result is qualitatively consistent with the result of the dissociation dynamics of $\mathrm{D}_{2} \mathrm{H}^{+}$mentioned above, where the dissociation to the $\mathrm{H}^{+}$channel is favored rather than that to one of the two $\mathrm{D}^{+}$channels at zero total angular momentum [4].

\section{B. Roles played by the averaged force field and reaction path potential}

The branching ratio of the dissociation dynamics of the modified $M_{3}$ cluster observed above is explained by the averaged force field in Sec. III. First, the reaction paths for dissociation are defined as the broken lines in Fig. 9 for respective channels. That is, along each dissociation path, the dissociating atom goes along the line perpendicular to the

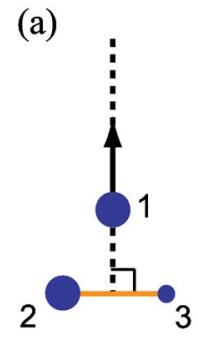

Channel 1

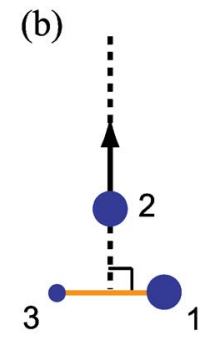

Channel 2

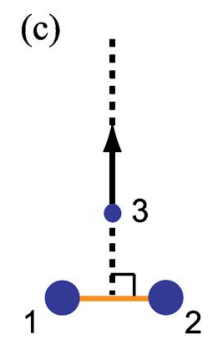

Channel 3
FIG. 9. (Color online) Classification of the dissociation channels of the modified $M_{3}$ cluster. The number of each channel is named by the label of the dissociating atom. Channel 1 and channel 2 are essentially equivalent, in which one of the heavy atoms dissociates. In channel 3, the light atom dissociates. The broken lines represent the dissociation paths of this study. 


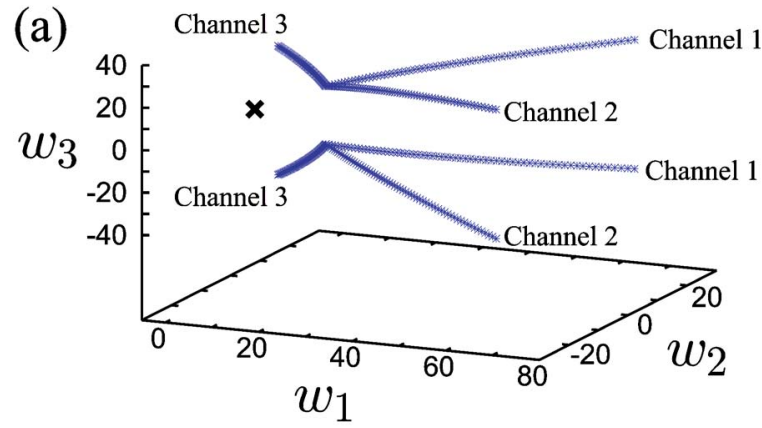

(b)

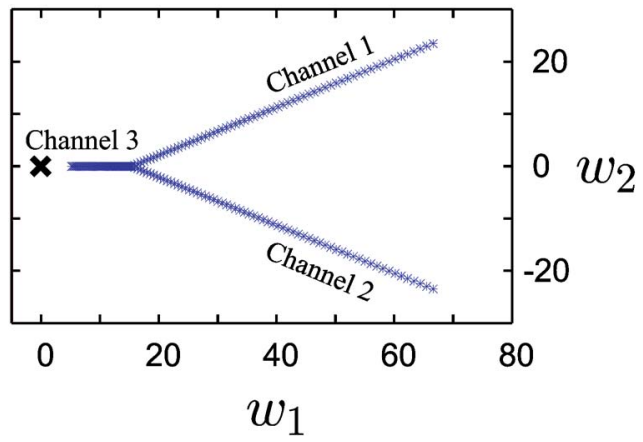

FIG. 10. (Color online) (a) Dissociation paths of the modified $M_{3}$ cluster in the internal space. The number of each dissociation channel is indicated in the figure. Cross mark represents the origin $\left(w_{1}, w_{2}, w_{3}\right)=(0,0,0)$. (b) is a top view of (a).

remaining diatomic bond. Each reaction path starts at the equilibrium configuration (equilateral triangle). Reaction paths thus defined can characterize overall directionality that dissociating trajectories in respective channels have in the internal space. (It should be noted that the diatomic system after dissociation can rotate. However, since rotation can be in either way, clockwise and counterclockwise, the above defined reaction paths can be regarded as rough averages of the dissociating trajectories.) Figure 10 shows the dissociation paths thus defined in the internal space. Since the cluster has two permutational isomers, each channel has two copies of dissociation path in the both sides of the $w_{1}-w_{2}$ plane. Each dissociation path extends to infinity.

Since the central force field as in Fig. 5 is effectively arising in the dynamics in the internal space, relationship between the directionality of each dissociation path and that of the central force field should be important in the branching process of the dissociation dynamics. It is evident that the dissociation paths for channel 1 and 2 go against the central force field since these paths get away from the origin. Therefore it is expected that the central force field works to prevent the dissociation reactions for channel 1 and channel 2. On the other hand, the path for dissociation channel 3 rolls up the origin as can be seen from Fig. 10(a). Therefore dissociation reaction in channel 3 should not be hindered very much compared with the reactions in channel 1 and 2 . These observations qualitatively explain the system's preference to dissociation channel 3 rather than channels 1 and 2 .

In order to quantify the above inspections, we compute the reaction-path potential in the same way as in Eq. (29) along each dissociation path in Fig. 10. Figures 11(a) and
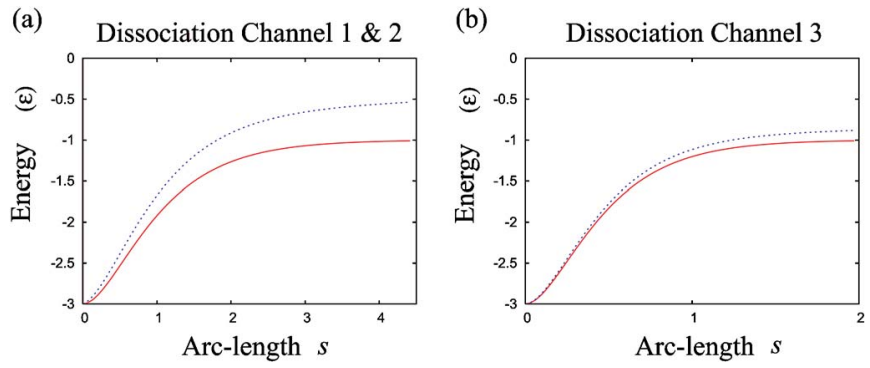

FIG. 11. (Color online) Potential energy (solid lines) and the reaction-path potential (broken lines) at $E=-0.5 \varepsilon$ along the dissociation paths in Fig. 10. (a) is for channels 1 and 2, and (b) is for channel 3.

11(b) show the original potential and the reaction-path potential along respective paths at total energy $E=-0.5 \varepsilon$. Original potential has the same barrier height for the two types of channels. On the other hand, it is now clear that the central force field acts as barrier more intensively for dissociation channel 1 and 2 making the barrier of the reactionpath potential for these channels higher than that for channel 3. We confirmed essentially the similar tendency of effective potential curves for other energy values. In this way, effect of the central force field is characterized for each dissociation channel.

\section{CONCLUDING REMARKS}

This paper has investigated, both numerically and analytically, how different atomic mass configurations affect branching ratios of multichannel reactions of a triatomic cluster. Atomic masses have been incorporated into internal coordinates and the associated non-Euclidean metric tensor after a correct separation of the rotational degrees of freedom. Because of the non-Euclidean nature of this metric tensor, terms including connection coefficients arise in the internal equations of motion, which serve as velocitydependent forces that one sees explicitly in a coordinate chart. By averaging these terms, we have deduced an averaged force field, which is a central force field in the $\left(w_{1}, w_{2}, w_{3}\right)$-coordinate chart. This force field accounts for statistical tendencies in the behavior of geodesics in the internal space and this plays a crucial role in determining the branching ratio of isomerization and the dissociation dynamics of the triatomic molecule. The effects of this averaged force field have been quantified by a reaction-path potential, which accounts for the mass-influenced branching ratios fairly well.

As has been demonstrated, the mass configuration itself can be an important factor to determine branching ratios of multichannel reactions. For example, mass configurations can even create a dynamical barrier as opposed to a potential barrier for certain channels such as the isomerization channel 3 and the dissociation channels 1 and 2 in this study. This kind of barrier related to the mass configurations may be responsible for various isotope effects. Since these massrelated barriers originate from the kinetic energy of the system, their influence is expected to be significant, especially 
in high-energy reactions. The result shown in Fig. 4 confirms this tendency, in which the bias in the reaction frequency becomes more significant in a higher energy range.

It is noteworthy that while the Born-Oppenheimer potential energy surface changes depending on molecule, the expression of the kinetic energy does not change if massweighted coordinates are employed. Concretely, the left-hand sides of Eqs. (18)-(20) are common to all triatomic systems with zero total angular momentum, although the potential function on right hand sides changes depending on the system. Therefore the averaged force field Eq. (27) introduced in the $\left(w_{1}, w_{2}, w_{3}\right)$-coordinate chart should be of rather universal importance in a variety of triatomic reactions. By comparing the directionality of an arbitrary reaction path and that of the averaged force field, which is the central force field pointing to the origin, one will be able to speculate whether the reaction path is favorable or not.

Here are some important next steps of the present work. For one thing, the use of microcanonical averaging of Eq. (22) for velocity-dependent forces could be modified. In the present study, the microcanonical averaging worked since the energy range of interest has been high and the dynamics of the system is expected to be sufficiently stochastic. Also, the quantities of interest in this work such as the reaction frequency and the branching ratio are the ones that are related to the long-time or the statistical properties of the system. On the other hand, in lower energy range or in a shorter time scale, invariant manifold tubes [48-52] and their intersection structures in phase space should be important in determining branching ratios. These phase-space structures may induce the breakdown of the equipartition law in short time dynamics. In such a situation, another methodology to characterize the phase space structure based on the breakdown of equipartition law may be useful [53]. Second, it might be ideal to get rid of the coordinate dependence of the present study along the lines of Ref. [42]. However, a good coordinate chart is often useful for the analysis of molecular reactions since it makes a "global" analysis of reaction dynamics possible, as in the present paper. Third, role of the mass configuration in systems with nonzero angular momentum is also an interesting issue. In a finite-angular-momentum system, velocity-dependent fictitious forces such as the centrifugal force and the Coriolis force also come into play in addition to the velocity-dependent force in this paper. The former two forces influence the internal dynamics of a molecule via the gauge fields in internal space [21], which are also closely related to atomic masses. These gauge fields can affect branching ratios and reaction rates.

To summarize, the key to elucidating the role of mass configurations in molecular reaction dynamics lies in the geometric structure of internal space. By analyzing those geometric structures carefully, one will ultimately be able to determine branching ratios of multichannel reactions at least qualitatively before computing a number of trajectories. An application of the present methodology to general molecular reactions, especially to isotopic reactions will be an important next step.

\section{ACKNOWLEDGMENTS}

The authors would like to thank Robert Littlejohn, Kevin Mitchell, and Kazuo Takatsuka for valuable discussions. The work was partially supported by NSF-ITR Grant No. ACI0204932, ICB-ARO Grant No. DAAD19-03-D-0004, and Grant No. NSF-DMS-0505711. One of the authors (T.Y.) has also been supported by JSPS.
[1] S. Datz, M. Larsson, C. Stromholm, G. Sundström, V. Zengin, H. Danared, A. Källberg, and M. af Ugglas, Phys. Rev. A 52, 2901 (1995).

[2] D. Strasser, L. Lammich, H. Kreckel, M. Lange, S. Krohn, D. Schwalm, A. Wolf, and D. Zajfman, Phys. Rev. A 69, 064702 (2004).

[3] C. M. Laperle, J. E. Mann, T. G. Clements, and R. E. Continetti, Phys. Rev. Lett. 93, 153202 (2004).

[4] M. Berblinger and C. Schlier, J. Chem. Phys. 101, 4750 (1994).

[5] K. Mauersberger, B. Erbacher, D. Krankowsky, J. Günther, and R. Nickel, Science 283, 370 (1999).

[6] B. C. Hathorn and R. A. Marcus, J. Chem. Phys. 111, 4087 (1999).

[7] Y. Q. Gao and R. A. Marcus, Science 293, 259 (2001).

[8] Y. Q. Gao and R. A. Marcus, J. Chem. Phys. 116, 137 (2002).

[9] S. Y. Grebenshchikov, R. Schinke, P. Fleurat-Lessard, and M. Joyeux, J. Chem. Phys. 119, 6512 (2003).

[10] D. Babikov, B. K. Kendrick, R. B. Walker, R. T. Pack, P. Fleurat-Lesard, and R. Schinke, J. Chem. Phys. 119, 2577 (2003); 119, 6298 (2003).

[11] M. V. Ivanov, S. Y. Grebenshchikov, and R. Schinke, J. Chem. Phys. 120, 10015 (2004).
[12] H. Akagi, Y. Fujimura, and O. Kajimoto, J. Chem. Phys. 110, 7264 (1999); 111, 115 (1999).

[13] J. I. Steinfeld, J. S. Francisco, and W. L. Hase, Chemical Kinetics and Dynamics (Prentice-Hall, Englewood Cliffs, NJ, 1989).

[14] R. A. Marcus, J. Chem. Phys. 45, 4493 (1966); 45, 4500 (1966); 49, 2610 (1968).

[15] F. T. Wall and R. N. Porter, J. Chem. Phys. 39, 3112 (1963).

[16] K. Takatsuka, H. Ushiyama, and A. Inoue-Ushiyama, Phys. Rep. 322, 347 (1999).

[17] W. H. Miller, N. C. Handy, and J. E. Adams, J. Chem. Phys. 72, 99 (1980).

[18] W. H. Miller, J. Chem. Phys. 76, 4904 (1982).

[19] C. Eckart, Phys. Rev. 47, 552 (1935).

[20] E. B. Wilson, Jr., J. C. Decius., and P. C. Cross, Molecular Vibrations (McGraw-Hill, New York, 1955).

[21] R. G. Littlejohn and M. Reinsch, Rev. Mod. Phys. 69, 213 (1997).

[22] T. Yanao and K. Takatsuka, J. Chem. Phys. 120, 8924 (2004).

[23] J. E. Marsden, T. S. Ratiu, and J. Scheurle, J. Math. Phys. 41, 3379 (2000).

[24] J. E. Marsden and T. S. Ratiu, Introduction to Mechanics and Symmetry (Springer, New York, 1999). 
[25] M. Kummer, Indiana Univ. Math. J. 30, 281 (1981).

[26] J. E. Marsden, R. Montgomery, and T. Ratiu, Contemp. Math. 28, 101 (1984).

[27] A. Guichardet, Ann. I.H.P. Phys. Theor. 40, 329 (1984).

[28] A. Tachibana and T. Iwai, Phys. Rev. A 33, 2262 (1986).

[29] T. Iwai, Ann. I.H.P. Phys. Theor. 47, 199 (1987); J. Math. Phys. 28, 964 (1987); Phys. Lett. A 162, 289 (1992).

[30] T. Iwai, J. Math. Phys. 28, 1315 (1987).

[31] F. T. Smith, J. Math. Phys. 3, 735 (1962).

[32] R. G. Littlejohn and M. Reinsch, Phys. Rev. A 52, 2035 (1995).

[33] R. G. Littlejohn, K. A. Mitchell, V. Aquilanti, and S. Cavalli, Phys. Rev. A 58, 3705 (1998).

[34] F. T. Smith, Phys. Rev. 120, 1058 (1960).

[35] W. Zickendraht, J. Math. Phys. 10, 30 (1969).

[36] R. T. Pack and G. A. Parker, J. Chem. Phys. 87, 3888 (1987).

[37] X. Chapuisat and A. Nauts, Phys. Rev. A 44, 1328 (1991).

[38] A. Kuppermann, J. Phys. Chem. 100, 2621 (1996).

[39] V. Aquilanti and S. Tonzani, J. Chem. Phys. 120, 4066 (2004).

[40] T. Yanao and K. Takatsuka, Phys. Rev. A 68, 032714 (2003).

[41] T. Yanao and K. Takatsuka, Adv. Chem. Phys. 130B, 87
(2005).

[42] H. Teramoto and K. Takatsuka, J. Chem. Phys. 122, 074101 (2005).

[43] T. L. Beck, D. M. Leiter, and R. S. Berry, J. Chem. Phys. 89, 1681 (1988).

[44] C. Amitrano and R. S. Berry, Phys. Rev. Lett. 68, 729 (1992).

[45] R. J. Hinde, R. S. Berry, and D. J. Wales, J. Chem. Phys. 96, 1376 (1992).

[46] R. J. Hinde and R. S. Berry, J. Chem. Phys. 99, 2942 (1992).

[47] E. Yurtsever, Phys. Rev. A 58, 377 (1993).

[48] N. De Leon, M. A. Mehta, and R. Q. Topper, J. Chem. Phys. 94, 8310 (1991).

[49] T. Uzer, C. Jaffé, J. Palacián, P. Yanguas, and S. Wiggins, Nonlinearity 15, 957 (2002).

[50] H. Waalkens, A. Burbanks, and S. Wiggins, J. Chem. Phys. 121, 6207 (2004).

[51] F. Gabern, W. S. Koon, J. E. Marsden, and S. D. Ross, Physica D 211, 395 (2005).

[52] C. B. Li, Y. Matsunaga, M. Toda, and T. Komatsuzaki, J. Chem. Phys. 123, 184301 (2005).

[53] T. Yanao, W. S. Koon, and J. E. Marsden (unpublished). 